\title{
Improvement in the mechanical properties of light curing epoxy resin with MFC (Micro-Fibrillated Cellulose)
}

\author{
Y. Ohnishi ${ }^{1}$, T. Fujii ${ }^{2} \&$ K. Okubo ${ }^{2}$ \\ ${ }^{1}$ Graduate Student of Doshisha University, Japan \\ ${ }^{2}$ Department of Mechanical Engineering and Systems, \\ Doshisha University, Japan
}

\begin{abstract}
This study shows an effective method to reinforce light curing epoxy resin with MFC (Micro-Fibrillated Cellulose) extracted from wood pulp. MFC is strongly hydrophilic and it is kept with water. Therefore, absorbed water in MFC was removed by two procedures (treatments): (1) freeze-drying and (2) substituting the water in MFC with ethanol. Absorbed water needs to be removed in order to reinforce the light curing resin with MFC because included water degrades the properties of the resin. The variation in the mechanical properties according to the type of treatment was examined after the composites were fabricated with the treated MFC. Debonding between MFC and the resin was observed in the freezedried MFC composite. On the other hand, breakage of MFC was observed in the ethanol-treated MFC composite. In order to evaluate the interfacial adhesion between MFC and the resin, the interfacial shear strength was defined by measuring the critical fiber length. When MFC was treated with ethanol, the interfacial shear strength between MFC and the resin increased 2.7 times as high as that of the freeze-dried MFC composite. In the SEM observation of the fracture surface of the ethanol-treated MFC composite, fiber bridging of MFC was observed. Fiber bridging prevented the crack propagation in the ethanoltreated MFC composite, and the energy absorption was improved by $58 \%$ in comparison with that of the pure resin.

Keywords: MFC (Micro-Fibrillated Cellulose), composite, fiber bridging, energy absorption, ethanol treatment, nanocomposite, light curing epoxy resin.
\end{abstract}




\section{Introduction}

Light curing resin is cured by an UV laser and is applied with the IH Process (Integrated Harden Polymer Process) [1,2]. The light curing resin has recently been focused on for application in the MEMS (Micro Electro Mechanical System) because fine micro-structures are available with the light curing resin in the IH Process [1,2]. In order to apply the light curing resin to structures in engineering applications, the mechanical properties of the resin should be improved against the problem of brittle fracture that is found under applied load. Fiber reinforcing is one effective method of improving the mechanical properties of resins as many engineers and researchers know. However, some problems were shown in fabricating the three-dimensional structure of a light curing resin with a fine shape by the IH Process. For example, it is difficult to align long fibers in the micro-structures by the IH process. When a conventional short fiber with a low aspect ratio was used as the reinforcement of the light curing resin, the failure strain was decreased in spite of improving the strength and elastic modulus of the resins. On the other hand, nano-materials improve the fracture toughness of resins without decreasing the strength and elastic modulus of the resins after they are well dispersed [3]. Carbon nano-tubes are well known as one of the most promising materials due to superior mechanical properties. However, for the current material, the hardening of the light curing resin was disturbed by the carbon nano-tube because the UV rays were intercepted due to the color being black.

In contrast, Micro Fibrillated Cellulose (MFC) is compatible as reinforcement with the light curing resin because the MFC has a clear color and extremely high aspect ratio with a nano-sized diameter. The refractive index of MFC to light is almost equal to that of the resin. In addition, the MFC has high mechanical properties such as approximately $2 \mathrm{GPa}$ and $140 \mathrm{GPa}$ of strength and Young's Modulus, respectively [4-6]. However, absorbed water in the MFC should be removed before mixing into the light curing resin because included water degrades the properties of the resin [7].

The purpose of this study is to improve the mechanical properties of light curing epoxy resin reinforced by MFC. In order to remove water from the MFC, two kinds of treatment were conducted, which are (1) freeze-drying and (2) substituting the water in the MFC with ethanol. The influence of the substituted ethanol on the resin was evaluated under several conditions of temperature where the ethanol in the resin was evaporated. The variation in the mechanical properties of the MFC reinforced epoxy composite due to the water removing treatment was well focused. The importance of the interfacial adhesion between MFC and the resin in the MFC composite was also established. The energy absorption was defined to evaluate the fracture toughness of the MFC composites. 


\section{Experiments}

\subsection{Materials}

Light curing epoxy resin (TSR820: CMET inc.) and commercial MFC (CELISH: Daicel chemical inc.) were used as the matrix and reinforcement of the current composite, respectively. Figure 1 shows the appearance and SEM picture of the MFC. The web-like network structure $[5,6]$ of the nano-scale is shown in figure 1.
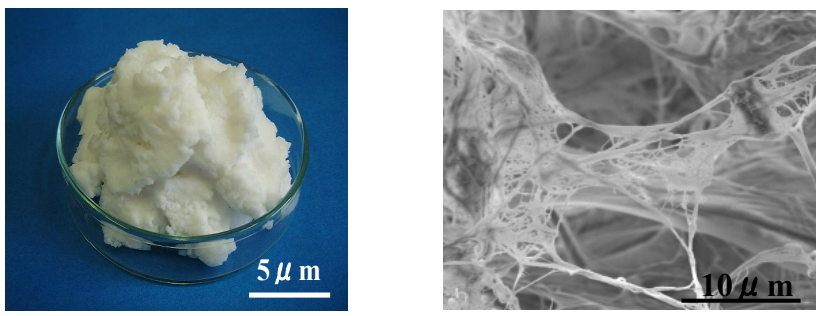

Figure 1: $\quad$ Micro-Fibrillated Cellulose.

\subsection{Freeze dry treatment for MFC}

Water in the MFC was removed with a freeze-dry machine (FDU-1100: TOKYO RIKAKIKAI Co.). The MFC treated by the freeze-drying process was referred to as "FD-MFC" in this study. The FD-MFC was mixed into the liquid resin, and dispersed in the homogenizer (PH91: SMT Co.) and the ultrasonic homogenizer (US-300T: NIHONSEIKI KAISHA LTD.).

\subsection{Ethanol treatment for MFC}

Figure 2 shows the substituted ratio of water included in the MFC by ethanol treatment. The substituted ratio was estimated by the curve, where the weight of ethanol to that of water was measured. $1 \mathrm{~g}$ of MFC (10 g CERISH, because MFC contains $90 \mathrm{wt} \%$ water) was used when the substituted ratio was measured.

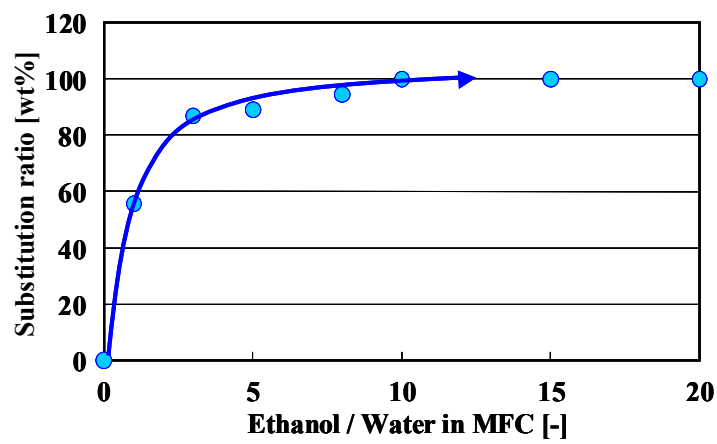

Figure 2: Substitution ratio of water in MFC to ethanol. 
The procedure to replace water in the MFC with ethanol is as follows. (1) Ethanol was purred to the MFC. In the process, firstly the measured ethanol was poured onto the MFC, where the weight of the ethanol was 15 times that of the water included in the MFC. (2) The solution including the MFC and the ethanol was mixed for 10 minutes and filtrated under $0.06 \mathrm{MPa}$. The MFC obtained by this process is referred as "ET-MFC" in this study. The ET-MFC was mixed with the resin and dispersed as described in section 2.1. Then, the mixed resin was heated at 80,90 or 100 degree $C$ in an oven to evaporate the ethanol in the resin.

\subsection{Fabrication procedure of specimens}

The resins prepared in sections 2.1 and 2.2 were cured to fabricate the test specimens by the conventional UV curing technique. The density of the radiated energy and the wavelength of the UV laser were $100 \mathrm{~mW} / \mathrm{cm}^{2}$ and $365 \mathrm{~nm}$, respectively.

Figure 3 shows the schematic diagram of the system of the IH Process, and figure 4 shows the geometry of the test specimens. Table 1 shows the type of the test specimens. The "Additive" in table 1 indicates the reinforcement of composites (ET-MFC and FD-MFC) and impurity (ethanol). The "Treatment temperature" in table 1 indicates the oven temperature used to evaporate the ethanol in the resins.

\subsection{Static tensile test}

A small capacity tensile testing machine (ASG-H/EZ test, Shimadzu Co.) was used and the test speed with stroke control was $1 \mathrm{~mm} / \mathrm{min}$. In this study, energy absorption is defined as the area in the stress-strain curve.

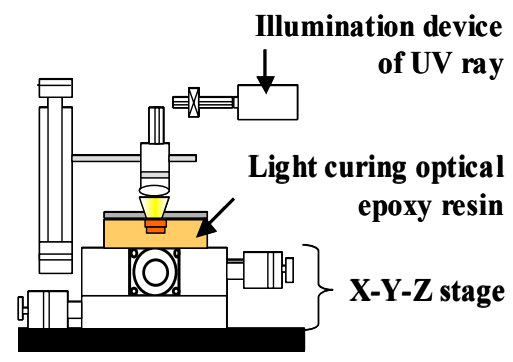

Figure 3: Integrated hardening polymer process.

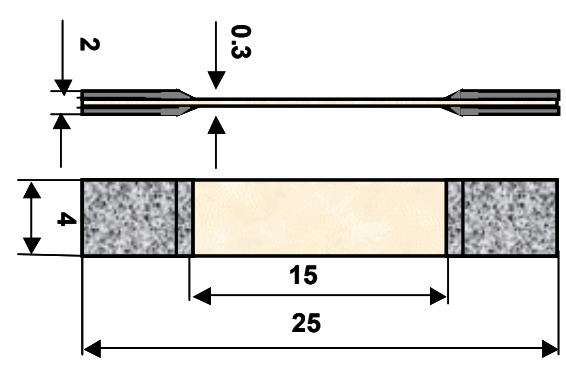

Figure 4: Geometry of the specimen (Each dimension is in "mm"). 
Table 1: $\quad$ Type of test specimens.

\begin{tabular}{c|c|c|c}
\hline Type & Additive & Treatment temperature [ $\left.{ }^{\circ} \mathrm{C}\right]$ & Content [wt\%] \\
\hline \hline \multirow{3}{*}{$\mathrm{A}$} & \multirow{3}{*}{ ET-MFC } & 80 & 0.5 \\
\cline { 3 - 4 } & & 90 & 0.5 \\
\cline { 3 - 4 } & & 100 & 0.5 \\
\hline \multirow{3}{*}{$\mathrm{B}$} & FD-MFC & - & 0.5 \\
\hline \multirow{3}{*}{$\mathrm{C}$} & \multirow{3}{*}{ Ethanol } & 70 & 50 \\
\cline { 3 - 4 } & & 80 & 50 \\
\cline { 3 - 4 } & & 90 & 50 \\
\cline { 3 - 4 } & & 100 & 50 \\
\hline
\end{tabular}

\section{Results and discussion}

\subsection{Influence from adding ethanol to light curing epoxy resin}

Figure 5 shows the tensile strength and Young's modulus of the specimen mixed ethanol in table 1 type $\mathrm{C}$. The mechanical properties of the specimens were almost constant by evaporating ethanol over 80 degree $\mathrm{C}$. The result revealed that the substituted ethanol did not affect the mechanical properties of the resin when the ethanol in the resin was evaporated at over 80 degree $\mathrm{C}$.

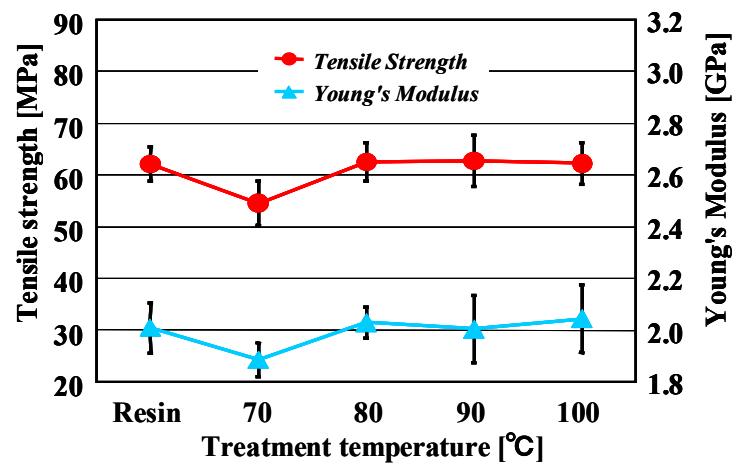

Figure 5: Influence of temperature on strength and Young's modulus of the light curing epoxy resin.

\subsection{Static tensile properties of MFC composites}

Figure 6 shows the stress-strain curves of specimens type A and B in table 1 . Table 2 summarizes the results of static tensile properties of the specimens. The tensile strength and Young's modulus of ET-MFC composites improved with the elevation of the treatment temperature. The tensile strengths of the ET-MFC composite treated at 90 and 100 degree $\mathrm{C}$ were $8 \%$ and $17 \%$ higher than that of the resin, respectively. On the other hand, the tensile strength decreased $6 \%$ compared to that of the resin when the material with ET-MFC was treated at 80 degree C. The Young's moduli of ET-MFC composites treated at 80 and 90 
degree $\mathrm{C}$ are almost the same as that of the resin. The Young's modulus of ETMFC composite treated at 100 degree $\mathrm{C}$ and of FD-MFC composite was improved $12 \%$ and $17 \%$, respectively, compared to that of the resin. The failure strain of the ET-MFC composite treated at 90 degree C increased remarkably. However, the failure strain decreased when the FD-MFC was used as the reinforcement.

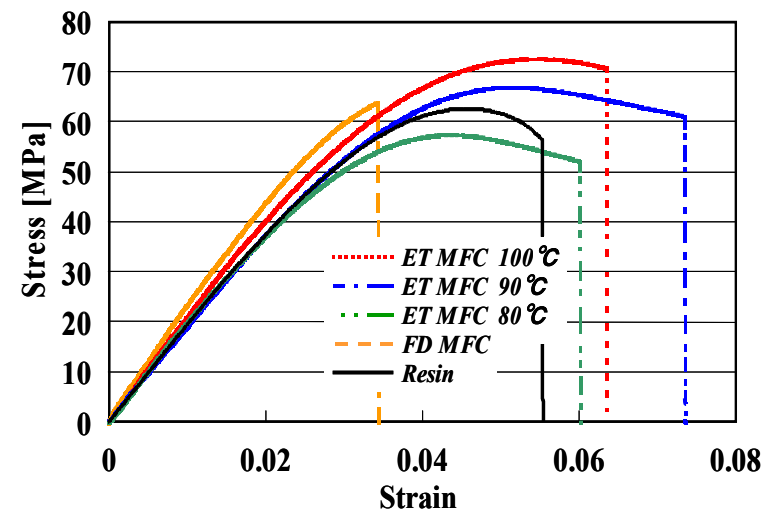

Figure 6: Stress-strain curves of the MFC composites.

Table 2: Mechanical properties of composites reinforced by ET-MFC and FD-MFC.

\begin{tabular}{l||c|c|c}
\hline & $\begin{array}{c}\text { Tensile Strength } \\
{[\text { MPa] }}\end{array}$ & $\begin{array}{c}\text { Young's Modulus } \\
\text { [GPa] }\end{array}$ & $\begin{array}{c}\text { Failure Strain } \\
{[\%]}\end{array}$ \\
\hline Resin & $62.1 \pm 1.7$ & $2.01 \pm 0.10$ & $5.54 \pm 0.43$ \\
\hline ET-MFC (80 degree C) & $58.6 \pm 1.8$ & $1.98 \pm 0.10$ & $6.01 \pm 0.14$ \\
\hline ET-MFC(90 degree C) & $67.1 \pm 2.7$ & $2.03 \pm 0.13$ & $6.93 \pm 0.36$ \\
\hline ET-MFC(100 degree C) & $72.7 \pm 3.5$ & $2.26 \pm 0.12$ & $6.49 \pm 0.30$ \\
\hline FD-MFC & $64.4 \pm 1.5$ & $2.36 \pm 0.14$ & $3.63 \pm 0.34$ \\
\hline
\end{tabular}

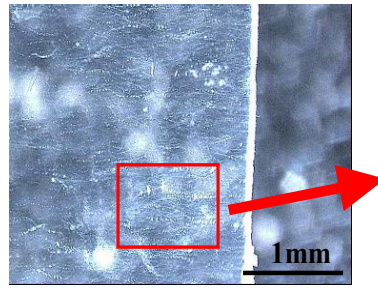

(a)

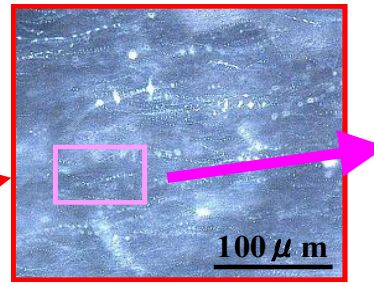

(b)

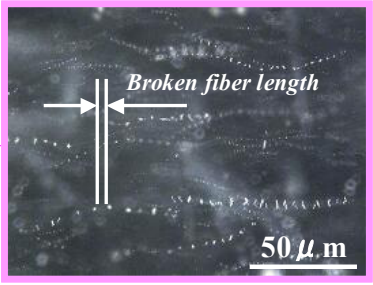

(c)

Figure 7: $\quad$ ET-MFC in the braking specimen. 
Figure 7 shows states of the failed ET-MFC composites observed by an optical microscope. A lot of broken points, appearing as white dots, were observed as shown in figure 7(a). Figure 7(b) and 7(c) show the local view of the square part in figure 7(a). It could be found that the white dots were observed only in the ET-MFC composite in which the MFC was treated with ethanol as shown in figure 7(b) and 7(c). In addition, the cracks might be propagated from the white dots in the ET-MFC near the fracture surface shown in figure 8. It was confirmed that the white crazing shown as white dots suggested the breakage of ET-MFC in the composite. These observations indicate that enough shear stress was sustained until failure of the specimen in the ET-MFC composites in which MFC was treated with ethanol.

However, in the FD-MFC composite, lots of interfacial debondings were observed between the MFC perpendicularly aligned to the tensile direction and the resin as shown in figure 9. It was considered that the failure strain in the FDMFC composite was decreased due to the debonding.

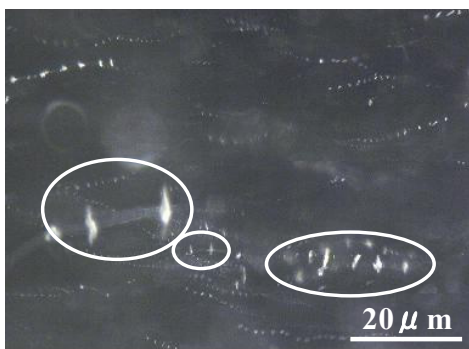

Figure 8: Crack propagation from breaking points of the ET-MFC.

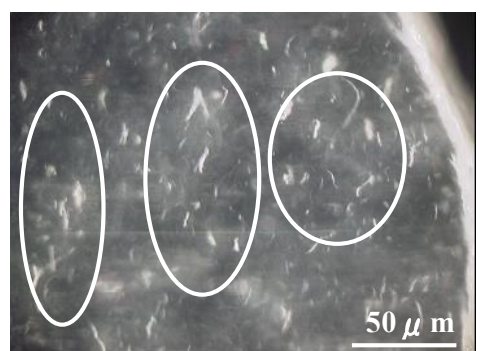

Figure 9: $\quad$ Interfacial debonding between the FD-MFC and the resin.

\subsection{Interfacial shear strength between MFC and light curing epoxy resin}

In this study, critical fiber length was defined as the broken fiber length of the MFC shown in figure 7(c). For clear observation of the breakage of MFC in the composites, in this session, the weight fraction ratio of the MFC (FD-MFC and ET-MFC) was set to $0.1 \mathrm{wt} \%$. Figure 10 shows the broken fiber length in each range of fiber-orientation angle; 0 5, 5 15, 15 25 and 25 35 degrees observed from the tensile direction (here, breakage of the FD-MFC could not be observed at 25 35 degrees). The broken fiber length increased if the MFC was aligned on the large off-set angle from the tensile direction. However, the broken fiber length decreased when the MFC was treated at a high treatment temperature. In this study, interfacial shear strength was calculated by the following equation [8].

$$
\begin{aligned}
\langle L\rangle & =\frac{3}{4} l_{c} \\
\frac{l_{c}}{d} & =\frac{\sigma_{f}}{2 \tau}
\end{aligned}
$$


Here, " $<\mathrm{L}>$ " denotes average broken fiber length in the range between $0 \sim 5$ degrees. The " $\sigma_{f}$ ", " $d$ " and " $1_{c}$ " denote the nominal tensile strength of MFC, the diameter of MFC and critical fiber length, respectively. In this study, " $\sigma_{\mathrm{f}}$ " and "d" were $2 \mathrm{GPa}$ and $300 \mathrm{~nm}$, respectively.

Figure 11 shows the interfacial shear strength calculated by the equation. The interfacial shear strength increased as the treatment temperature was elevated as shown in figure 12. On the other hand, the interfacial shear strength between FDMFC and the resin was approximately $11 \mathrm{MPa}$, which was $60 \%$ lower than that between ET-MFC and the resin at 100 degree $\mathrm{C}$ treatment. These results showed that an improvement of 2.7 times was obtained in interfacial shear strength between MFC and the resin when the MFC was treated with ethanol, compared to that by freeze-drying treatment.

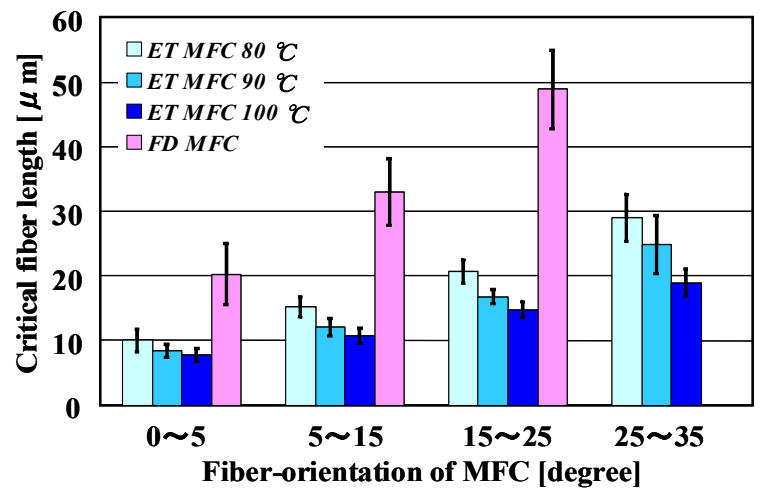

Figure 10: Broken fiber length with each fiber orientation angle.

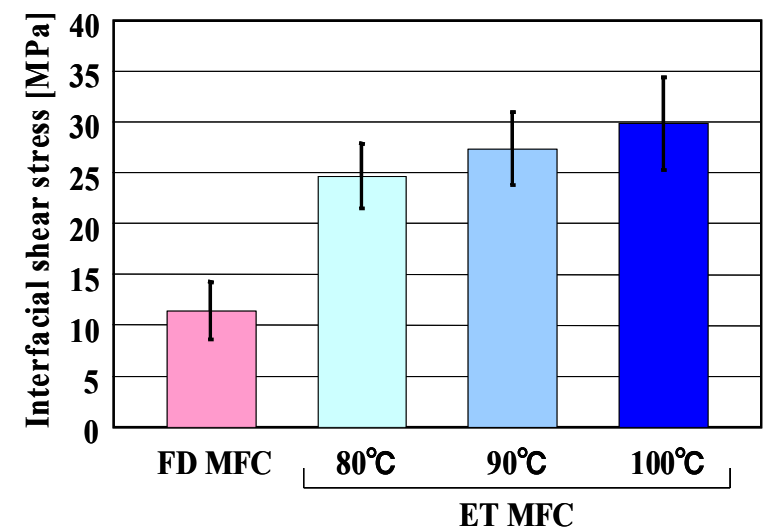

Figure 11: Interfacial shear strength of reinforced composites ET-MFC and FD-MFC. 


\subsection{Energy absorption of composites reinforced by MFC}

Figure 12 shows the energy absorption of type A and B of the FD-MFC composite and the ET-MFC composites, respectively, in table 1. The energy absorption until failure of the FD-MFC composite decreased to $54 \%$ of that of the resin. The interfacial debondings shown in figure 9 suggested that the energy absorption was decreased by poor interfacial adhesion between the FD-MFC and the resin. On the other hand, when MFC was treated with ethanol at 90 degree $\mathrm{C}$, the energy absorption increased 58\% compared to that of the resin in which measured energy absorption was largest among all data investigated in this study.

The fracture surface of the composite treated at 90 degree $\mathrm{C}$ is shown in figure 13. The fracture surface shows fiber bridging by addition of the MFC. This picture indicates that the fiber bridging prevented crack propagation in the ET MFC composite as explained in figure 14. This study proposed that the reinforcing MFC should be treated by ethanol to improve the mechanical properties of the composite of light curing epoxy resin.

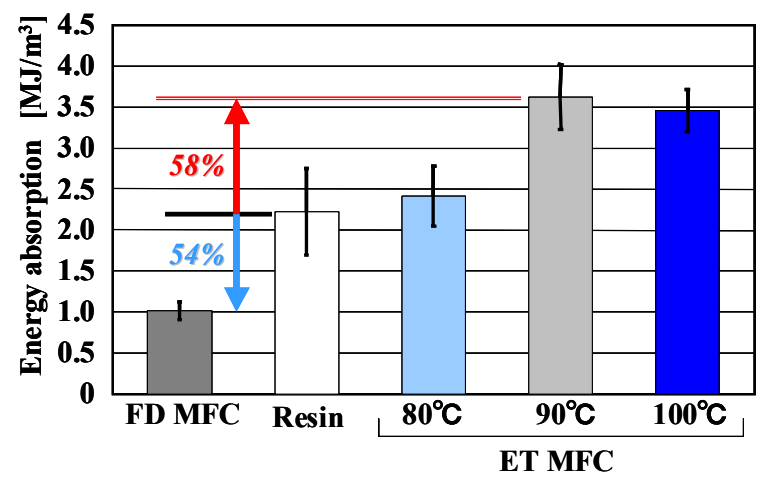

Figure 12: Energy absorption of composites reinforced by ET-MFC and FDMFC.

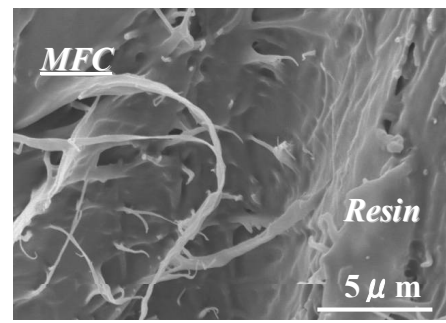

Figure 13: Fracture surface of composites reinforced by ET-MFC (treatment: $90^{\circ} \mathrm{C}$, contents: $\left.0.5 \mathrm{wt} \%\right)$. 


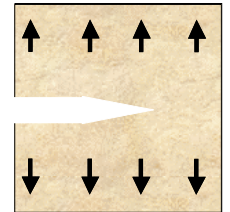

(a) Resin

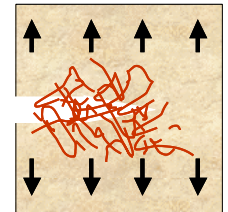

(b) ET MFC composite

Figure 14: Schematic model of fiber bridging by entangled MFC.

\section{Conclusion}

Some conclusions could be obtained as follows.

(1) Ethanol did not affect the mechanical properties of light curing epoxy resin when the ethanol in the resin was evaporated over 80 degree $C$.

(2) When the MFC was treated with ethanol, an improvement of 2.7 times was obtained in the interfacial shear strength between MFC and the resin, compared to that by freeze-drying treatment.

(3) Energy absorption of the MFC composite increased 58\% compared to that of light curing epoxy resin when the MFC was treated with the ethanol treatment.

\section{Reference}

[1] Takagi T, Nakajima N, "Photoforming Applied to Fine Forming." JSME Int Journal. Ser C. Mech Systems, 38-4, pp. 811-817, 1995.12

[2] Mcmains Sara,"Layered Manufacturing Technologies", Assoc Comput Mach, 48-6, pp. 50-56 (2005.06)

[3] B. Qi, Q. X. Zhang, M. Bannister, Y.-W. Mai, "Investigation of the mechanical properties of DGEBA-based epoxy resin with nanoclay additives", Composite Structures, 75, pp. 514-519 (2006)

[4] H. Yano, S. Nkahara, "Bio-composites produced from plant microfiber bundles with a nanometer unit web-like network", Journal of Materials science, 39, pp. 1635-1638 (2004)

[5] A. N. Nakagaito, H. Yano, "Novel high-strength biocomposites based on microfibrillated cellulose having nano-order-unit web-like network structure", Appl. Phys. A (2003).

[6] A. N. Nakagaito, S. Iwamoto, H. Yano, "Bacterial cellulose: the ultimate nano-scalar cellulose morphology for the production of high-strength composites", Appl. Phys. A (2004)

[7] Herbert H. Espy, "The mechanism of wet-strength development in paper-a review" Tappi Journal Vol. 78 No. 4 (1995)

[8] Takehito Fukuda, Zenichiro Maekawa, Toru Fujii, "Advances in Fiber Composite Materials" Current Japanese Materials Research Vol. 12, pp. 4345 (1994). 